\title{
Stereotactic probability and variability of speech arrest and anomia sites during stimulation mapping of the language dominant hemisphere
}

\author{
*Edward F. Chang, MD,1-4 Jonathan D. Breshears, MD, ${ }^{1}$ Kunal P. Raygor, MD, ${ }^{1}$ Darryl Lau, MD, ${ }^{1}$ \\ Annette M. Molinaro, PhD, ${ }^{1,5}$ and Mitchel S. Berger, MD' \\ Departments of ${ }^{1}$ Neurological Surgery, ${ }^{2}$ Physiology, and ${ }^{5}$ Epidemiology and Biostatistics; ${ }^{3}$ Center for Integrative Neuroscience, \\ University of California, San Francisco; and ${ }^{4}$ Center for Neural Engineering and Prostheses, University of California, Berkeley, \\ and University of California, San Francisco, California
}

OBJECTIVE Functional mapping using direct cortical stimulation is the gold standard for the prevention of postoperative
morbidity during resective surgery in dominant-hemisphere perisylvian regions. Its role is necessitated by the significant
interindividual variability that has been observed for essential language sites. The aim in this study was to determine
the statistical probability distribution of eliciting aphasic errors for any given stereotactically based cortical position in a
patient cohort and to quantify the variability at each cortical site.
METHODS Patients undergoing awake craniotomy for dominant-hemisphere primary brain tumor resection between
1999 and 2014 at the authors' institution were included in this study, which included counting and picture-naming tasks
during dense speech mapping via cortical stimulation. Positive and negative stimulation sites were collected using an
intraoperative frameless stereotactic neuronavigation system and were converted to Montreal Neurological Institute
coordinates. Data were iteratively resampled to create mean and standard deviation probability maps for speech arrest
and anomia. Patients were divided into groups with a "classic" or an "atypical" location of speech function, based on the
resultant probability maps. Patient and clinical factors were then assessed for their association with an atypical location
of speech sites by univariate and multivariate analysis.

RESULTS Across 102 patients undergoing speech mapping, the overall probabilities of speech arrest and anomia were 0.51 and 0.33 , respectively. Speech arrest was most likely to occur with stimulation of the posterior inferior frontal gyrus (maximum probability from individual bin $=0.025$ ), and variance was highest in the dorsal premotor cortex and the posterior superior temporal gyrus. In contrast, stimulation within the posterior perisylvian cortex resulted in the maximum mean probability of anomia (maximum probability $=0.012$ ), with large variance in the regions surrounding the posterior superior temporal gyrus, including the posterior middle temporal, angular, and supramarginal gyri. Patients with atypical speech localization were far more likely to have tumors in canonical Broca's or Wernicke's areas (OR $7.21,95 \% \mathrm{Cl}$ $1.67-31.09, p<0.01$ ) or to have multilobar tumors (OR 12.58, 95\% Cl 2.22-71.42, $p<0.01$ ), than were patients with classic speech localization.

CONCLUSIONS This study provides statistical probability distribution maps for aphasic errors during cortical stimulation mapping in a patient cohort. Thus, the authors provide an expected probability of inducing speech arrest and anomia from specific $10-\mathrm{mm}^{2}$ cortical bins in an individual patient. In addition, they highlight key regions of interindividual mapping variability that should be considered preoperatively. They believe these results will aid surgeons in their preoperative planning of eloquent cortex resection.

http://thejns.org/doi/abs/10.3171/2015.10.JNS151087

KEY WORDS language mapping; cortical stimulation; anomia; speech arrest; probability maps; functional neurosurgery

ABBREVIATIONS MNI = Montreal Neurological Institute; $\mathrm{PSTG}=$ posterior superior temporal gyrus; $\mathrm{VPMC}=$ ventral premotor cortex. SUBMITTED May 19, 2015. ACCEPTED October 28, 2015.

INCLUDE WHEN CITING Published online February 19, 2016; DOI: 10.3171/2015.10.JNS151087.

* Drs. Chang and Breshears contributed equally to this paper. 
$\mathrm{F}$ UNCTIONAL mapping using electrocortical stimulation has become the gold standard for the prevention of postoperative morbidity during surgery in the dominant hemisphere. This technique can be used to identify the cortical representation of motor, sensory, and language function and has been shown to increase rates of gross-total resection while limiting the number of latestage neurological deficits. ${ }^{1-4,6,7,22}$ Individualized mapping is especially important for identifying cortical speech arrest and anomia areas, which are highly variable among patients. This variability makes it difficult to preoperatively estimate the probability that a planned resection will result in an aphasic deficit.

Interindividual variability in the localization of speech arrest and anomia sites has been demonstrated in numerous cortical mapping studies, both intraoperatively and extraoperatively. ${ }^{5,10-13,17,18}$ Wilder Penfield was the first to systematically use direct cortical stimulation to determine essential language sites, and he specifically showed that midfrontal (outside of the canonical Broca's area) and midparietal areas could also be involved in speech production. ${ }^{14}$ Large, modern speech mapping series have all re-demonstrated that naming function can lie in the frontal, temporal, and/or parietal cortices, with the majority of anomia errors occurring in the posterior frontal and temporal cortices..$^{10,12,17,19}$ A number of patients in these series did not produce errors with stimulation of the classic Broca's area, further highlighting the variability of cortical language representation among individuals. . $^{5,10,13,17}$

While previous studies have described speech probability maps, the degree of interindividual variability at a particular cortical location directly affects the certainty or confidence interval associated with these probability estimates. Cortical areas with greater variability in speech representation across individuals have less certain probability estimates. We believe knowledge of both probability and variability is important in preoperative planning for resections adjacent to and involving potentially eloquent cortex. Further, previously reported probabilistic mapping results have been spatially resolved to the level of anatomical gyri or large cortical areas, while stereotactically derived probability maps have not been described. The accuracy of stereotactic measurements is more precise, and these values can be more easily compared across patients and with standard imaging studies such as functional MRI. Finally, most previous studies have not examined the effect of tumor or lesion location on the variability in spatial localization.

In this study, we had 3 goals: 1) to determine the stereotactically defined spatial probability distribution for eliciting anomia or speech arrest errors upon stimulation, 2) to determine the patterns of interindividual variability in spatial localization of essential language sites, and 3) to determine if any patient or lesion location factors correspond to patterns of greater variability.

\section{Methods}

\section{Study Subjects}

This study, which was approved by the Committee on
Human Research at the University of California, San Francisco, included patients undergoing awake craniotomy for dominant-hemisphere primary brain tumor resection at our institution between 1999 and 2014. One hundred three consecutive patients underwent surgery performed by the senior author (M.S.B.) between 1999 and 2005, and 9 patients underwent surgery performed by another author (E.F.C.) between 2011 and 2014. Ten patients were excluded based on the exclusion criteria of gross cortical dysplasia, significant mass effect and/or cortical distortion from the underlying lesion, insufficient extent or density of mapping, and lack of follow-up information in medical records.

\section{Intraoperative Language Mapping}

After exposure of the peri-rolandic cortex and emergence from intravenous sedation (either dexmedetomidine or propofol), intravenous fentanyl was titrated for optimal balance of pain control with patient arousal during the mapping procedure. Exposed cortex was completely and densely mapped by a neurosurgeon (M.S.B. or E.F.C.) using an Ojemann stimulator (current range 1-3.5 mA, pulse frequency $60 \mathrm{~Hz}$, pulse width $1 \mathrm{msec}$, stimulus duration 500-1500 msec, stimulator electrode spacing $5 \mathrm{~mm}$ ). Prior to mapping, the afterdischarge threshold was determined, and the mapping was conducted at the maximum current that did not result in cortical spread (that is, afterdischarges), which ensured a low false-negative rate. Each response or nonresponse to stimulation was tested for consistency and repeatability with at least 3 nonconsecutive stimulations. Responses were considered valid only in the absence of afterdischarges or seizure activity on electrocorticography, which was monitored and reported in real time by an epileptologist.

Each subject's brain was densely mapped for motor, sensory, and language function using a standardized protocol. Language function was mapped using 2 paradigms. First, intermittent stimulation was used during counting and/or recitation of the days of the week to test for speech arrest sites. Patients were instructed to count to 30 at a constant pace. Stimulation was applied following a normal verbalization without stimulation, just prior to subsequent verbalization. Cortical sites were stimulated sequentially, and the same site was not stimulated twice in a row. Anomia was similarly tested with a naming task in which patients viewed a monitor at a distance of 3 feet and named sequentially displayed pictures of common objects (horse, hammer, keyboard, and so forth). The pictures changed at regular intervals, and patients were instructed to name immediately at picture onset. The neurosurgeon (M.S.B. or E.F.C.) intermittently performed stimulation just prior to picture onset, and those sites that reproducibly caused an inability to name objects were labeled as positive for anomia. As with speech arrest mapping, cortical sites were tested only after a normal naming trail without stimulation, and the same cortical site was not tested twice in a row.

After the completion of mapping, photographs were taken to document cortical sites (Fig. 1A). Each stimulated cortical site (speech arrest, anomia, motor, sensory, and negative) was also captured using the BrainLab intraoperative neuronavigation software, which allowed the cortical 
A

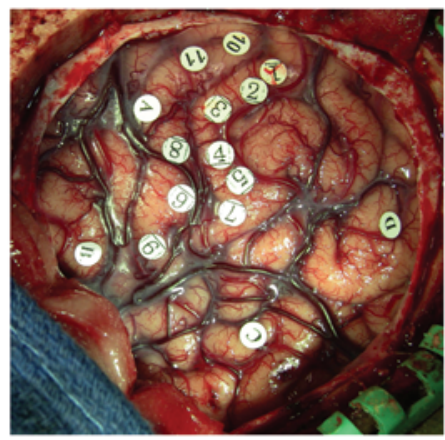

\section{B}
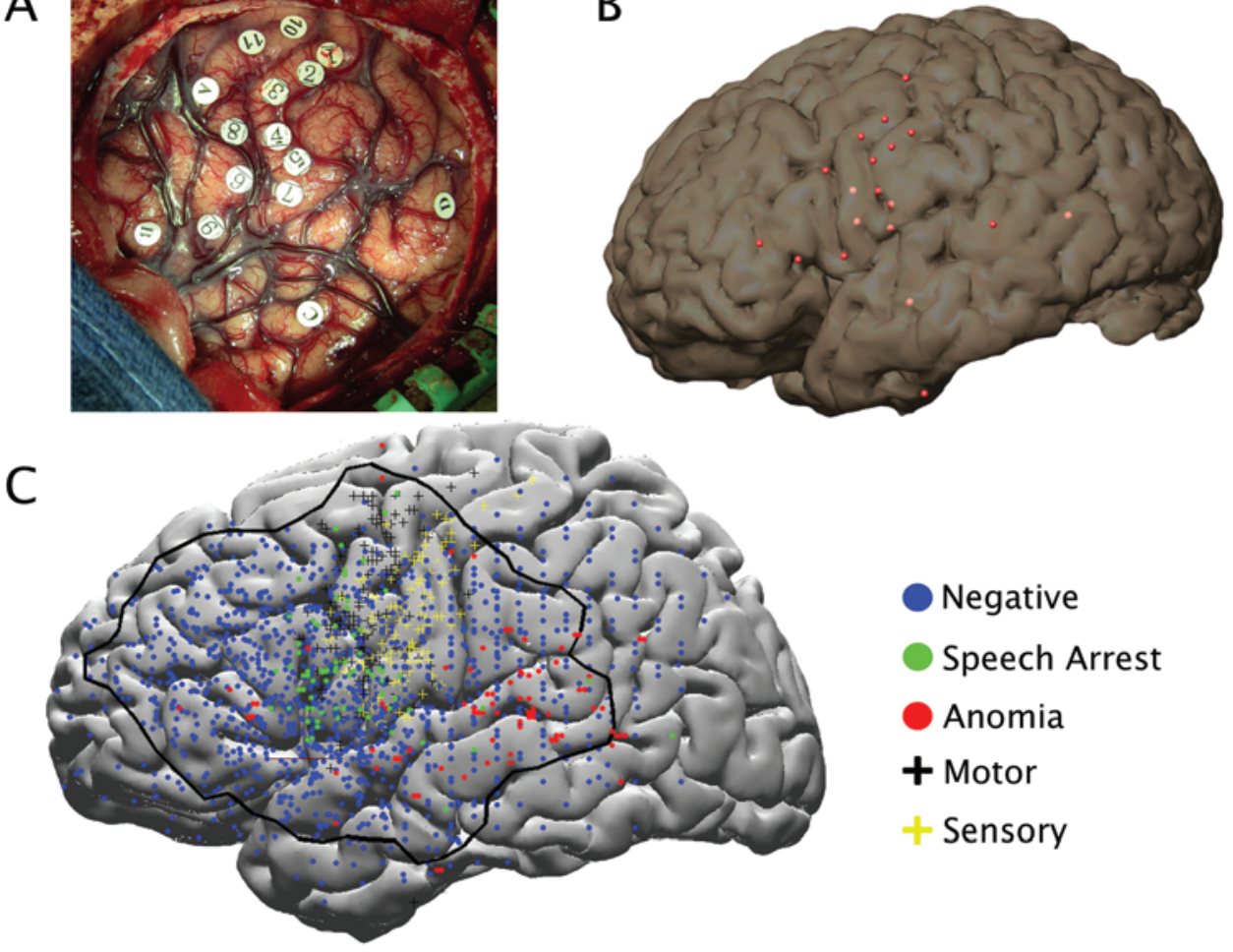

Negative

- Speech Arrest

Anomia

+ Motor

Sensory

D
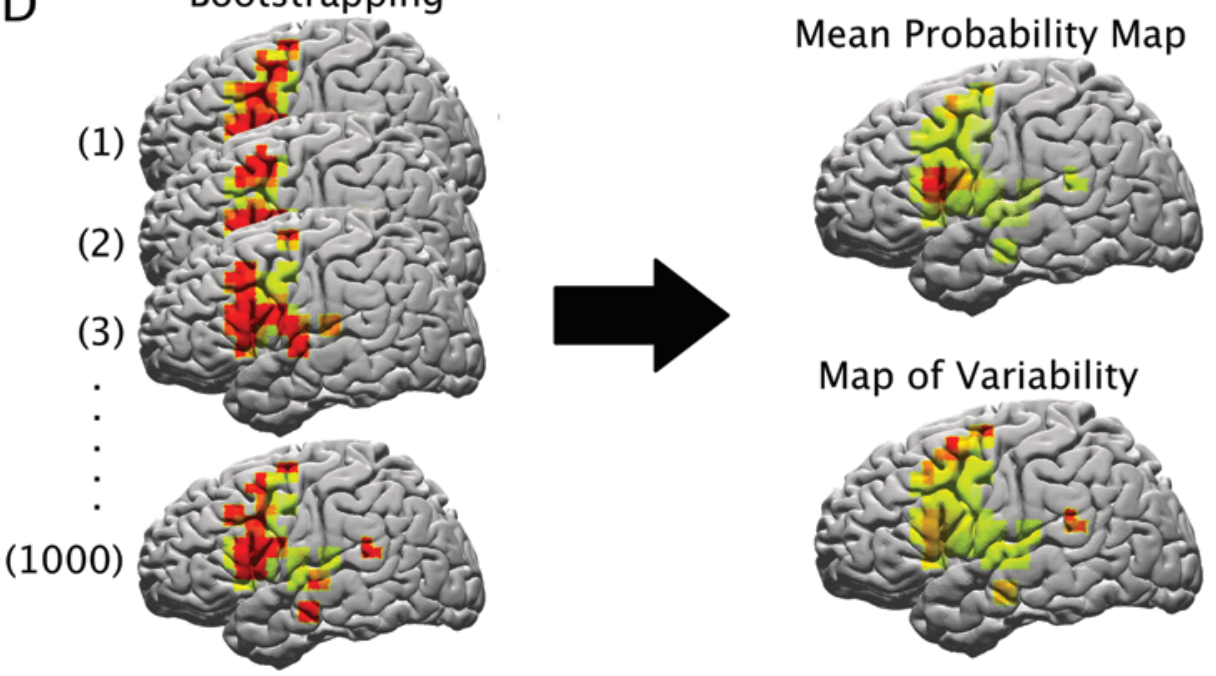

FIG. 1. A: Intraoperative photograph showing positive motor, sensory, speech arrest, and anomia sites after the completion of mapping. B: BrainLab MRI surface reconstruction showing stimulation sites from those marked in panel A. Negative sites were not included for visualization purposes. C: Montreal Neurological Institute brain template with stimulation sites compiled from all subjects. Black line encompasses the cortical area meeting the threshold of 10 stimulated sites per $10-\mathrm{mm}^{2}$ bin; the area outside this black line was not included in the analysis. D: Illustration of bootstrapping technique. Data from the MNI brain template in panel $\mathrm{C}$ was iteratively resampled with replacement to generate 1000 maps from which mean probability and variability could be calculated. Figure is available in color online only.

sites to be visualized on the surface reconstruction of the patient's brain MRI (Fig. 1B). It also allowed extraction of the 3D Montreal Neurological Institute (MNI) coordinates of each cortical site.

\section{Calculation of Probability and Variability}

The stimulated cortical sites from all patients were binned in a 2D histogram according to their y (anterior- posterior) and z (dorsal-ventral) MNI coordinates. Cortical bins containing fewer than 10 stimulations were excluded from further analysis (Fig. 1C). A sliding bin size of $10 \mathrm{~mm}^{2}$ with a $5-\mathrm{mm}$ overlap was selected. This spatial resolution choice was based on the 5-mm spacing of the Ojemann stimulator probe and an estimated 2- to 3-mm error of cortical site marking and MRI coregistration. This decision was further influenced by practical surgical 
considerations such as the typical gyrus width (approximately $10 \mathrm{~mm}$ ) and prior observations that a distance $>1$ $\mathrm{cm}$ from the resection margins and eloquent cortex minimizes permanent postoperative deficits. ${ }^{8}$

To quantify the probability of obtaining a speech arrest or anomia response at a particular cortical location, as well as to identify and quantify areas of increased interindividual variability, a bootstrapping technique was employed (Fig. 1D). For a given $10-\mathrm{mm}^{2}$ cortical bin, the probability of anomia (or speech arrest) is equal to the number of anomia sites in that bin divided by the total number of stimulated sites in the bin (anomia sites + sites negative for anomia). The data were iteratively resampled with replacement (1000 iterations) to generate 1000 probability maps from the data. These maps were normalized to reflect the observed probability of speech arrest or anomia in the aggregate data. A mean overall probability map was calculated by averaging across the 1000 bootstrapped maps. A map of the standard deviation of the probability, which reflects areas of increased interindividual variability, was also calculated from the 1000 bootstrapped maps. These maps of the probability and standard deviation (variability) of cortical speech arrest and anomia sites were then transferred onto a standard MNI brain template for visualization purposes. This analysis was performed using MATLAB.

\section{Predictors of Highly Variable Speech Sites}

Based on results of the probability and variability analysis, patients were classified as having either "classic" (if either speech arrest or anomia sites were localized in areas of low variability) or "atypical" (if sites were located in areas of high variability) speech localization. Classic localization for speech arrest included areas within the pars opercularis and pars triangularis, and for anomia included the posterior superior temporal gyrus (pSTG). Patients with language sites falling outside these areas were defined as having atypical localization. Associations between various patient and/or tumor characteristics and speech localization (classic or atypical) were determined by univariate analyses using either chi-square or MannWhitney U-test for categorical and continuous variables, respectively. Variables deemed to have an association with an atypical speech site location on univariate analysis $(\mathrm{p}<$ 0.2 ) were included in stepwise multivariate logistic regression with backward elimination. Odds ratios are reported with $95 \%$ confidence intervals, and the threshold for statistical significance was set at $p<0.05$. All statistical analyses were performed with SPSS version 22 (IBM Corp.).

\section{Results}

A total of 1768 cortical sites were stimulated across the 102 patients included in the study. The study population consisted of 60 men and 42 women, with a median age of 41 years (range 14-84 years). Tumors were located in the left and right hemisphere in 98 and 4 patients, respectively. The overall breakdown of tumor location by lobe was 20 frontal, 29 temporal, 11 parietal, 29 insular, and 13 multiple lobes. Additional demographic and clinical information is shown in Table 1. Speech arrest was identified at 69
TABLE 1. Summary of patient and tumor characteristics

\begin{tabular}{|c|c|}
\hline Variable & Value \\
\hline Median age in yrs (range) & $41(14-84)$ \\
\hline \multicolumn{2}{|l|}{ Sex, no. $(\%)$} \\
\hline Male & $60(59)$ \\
\hline Female & $42(41)$ \\
\hline \multicolumn{2}{|l|}{ Tumor hemisphere, no. (\%) } \\
\hline Lt & $98(96)$ \\
\hline $\mathrm{Rt}$ & $4(4)$ \\
\hline \multicolumn{2}{|l|}{ Tumor lobe, no. (\%) } \\
\hline Frontal only & $20(20)$ \\
\hline Temporal only & $29(28)$ \\
\hline Parietal only & $11(11)$ \\
\hline Insula only & $29(28)$ \\
\hline Multiple lobes & $13(13)$ \\
\hline Mean tumor vol in $\mathrm{cm}^{3}$ ( \pm SEM) & $46.91( \pm 6.6)$ \\
\hline \multicolumn{2}{|l|}{ Tumor pathology, WHO Grade, no. (\%) } \\
\hline Glioblastoma, IV & $22(22)$ \\
\hline Anaplastic astrocytoma, III & $26(25)$ \\
\hline Anaplastic oligodendroglioma, III & $4(4)$ \\
\hline Anaplastic oligoastrocytoma, III & $7(7)$ \\
\hline Oligodendroglioma, II & $16(16)$ \\
\hline Oligoastrocytoma, II & $10(10)$ \\
\hline Astrocytoma, I or II & $9(9)$ \\
\hline Primitive neuroectodermal tumor, IV & $2(2)$ \\
\hline Other, ${ }^{*}$ I or II & $6(6)$ \\
\hline \multicolumn{2}{|l|}{ WHO tumor grade, no. (\%) } \\
\hline I & $5(5)$ \\
\hline$\|$ & $35(34)$ \\
\hline III & $36(35)$ \\
\hline IV & $26(25)$ \\
\hline Preop seizure history, no. (\%) & $69(68)$ \\
\hline Preop AED use, no. (\%) & $68(67)$ \\
\hline
\end{tabular}

$\mathrm{AED}=$ antiepileptic drug; $\mathrm{SEM}=$ standard error of the mean.

* Other tumor pathologies include WHO Grade II ependymoma (1), WHO Grade I ganglioglioma (4), and WHO Grade I dysembryoplastic neuroepithelial tumor (1).

cortical sites across 52 subjects (51\% overall probability; Fig. 2). The median number of speech arrest sites among all 102 patients was 1 (range $0-4$ sites). The pars opercularis and precentral gyrus had the highest probability of speech arrest (maximum bin 2.5\%). The greatest variability in speech arrest was found in the dorsal premotor cortex and pSTG (SD 0.9\%). Anomia was identified at 94 cortical sites across 34 subjects (33\% overall probability; Fig. $3)$. Among all patients, the median number of anomia sites was 0 (range $0-8$ sites); however, when considering those with at least 1 positive anomia site, the median number of such sites was 2 (range 1-8 sites). The sites of maximal probability for anomia included the posterior perisylvian cortex, especially the pSTG (maximum bin 1.2\%). The areas surrounding the pSTG, including the posterior middle temporal, angular, and supramarginal gyri, showed the highest variability (SD 0.4\%), though anterior temporal stimulation sites also proved highly variable. Additionally, there were 187 motor sites, 127 sensory sites, and 1293 "negative" sites with no observed function. 

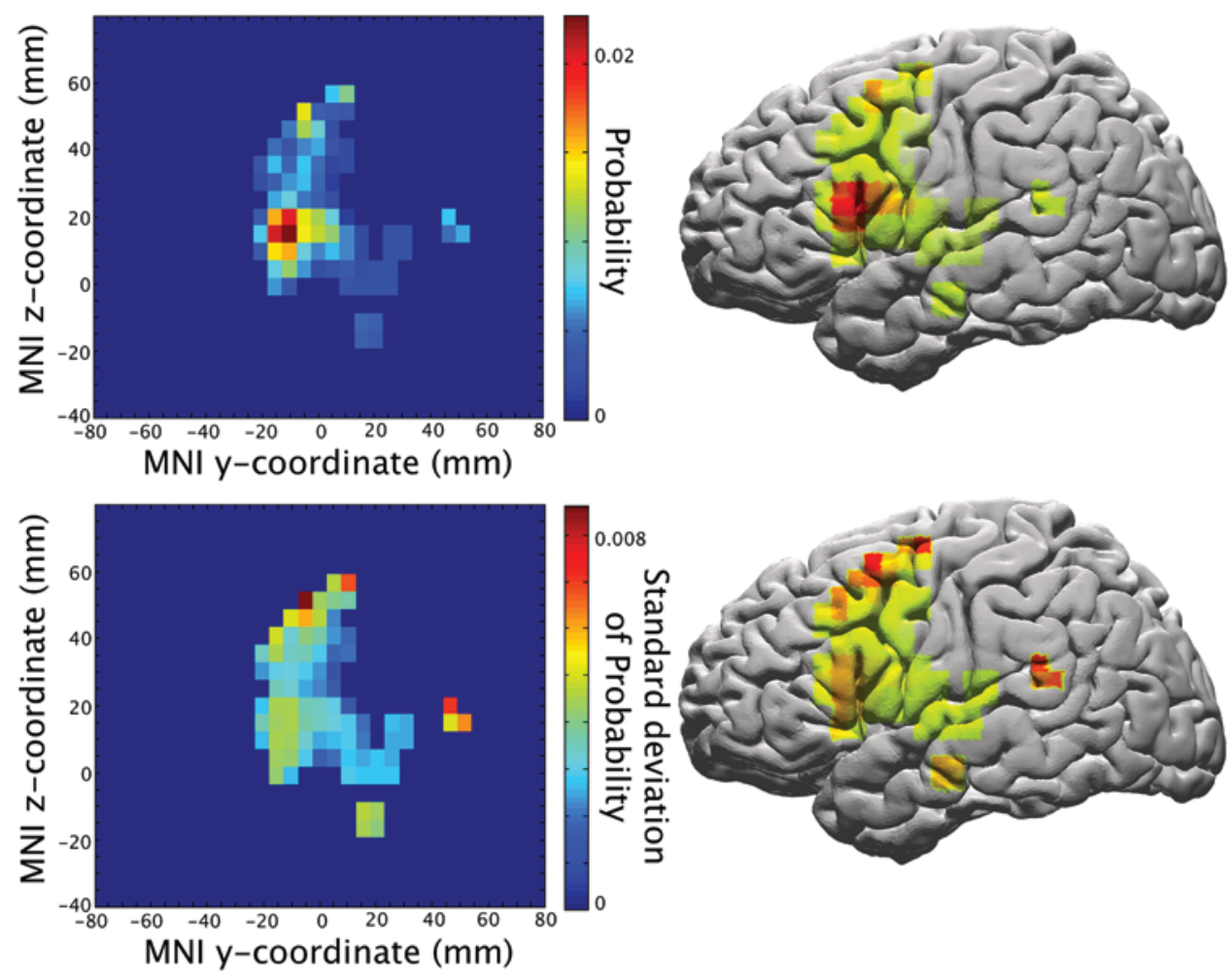

FIG. 2. Probability (upper) and variability (lower) of speech arrest sites. The posterior inferior frontal gyrus (pars opercularis or precentral gyrus) showed the highest probability of speech arrest, while the dorsal premotor cortex and pSTG demonstrated the greatest variability in speech arrest sites. Figure is available in color online only.

Eight patients had speech arrest sites distant from the posterior inferior frontal gyrus (pIFG; 6 in the dorsal premotor cortex and 2 in the pSTG), and 8 patients had anomia sites located in the posterior middle temporal, angular, or supramarginal gyrus. To determine possible predictors of atypical speech sites, patients were stratified across multiple variables of interest. Seven variables that demonstrated a possible association with the outcome of interest on univariate analysis $(\mathrm{p}<0.2)$ were included in the multivariate logistic regression. Variables that did not meet the threshold for inclusion in the multivariate analysis included age, sex, hemisphere of surgery, tumor volume, tumor grade, history of seizure, and preoperative use of various antiepileptic drugs (AEDs; carbamazepine, oxcarbazepine, lamotrigine, topiramate, felbamate, or any AED; Table 2). Compared with patients with speech sites in classic areas, the 16 patients with atypical speech sites were more likely to have tumors in the canonical Broca's or Wernicke's areas (OR 7.21, 95\% CI 1.67-31.09, p < 0.01). They were also more likely to have multilobar tumors (OR $12.58,95 \%$ CI $2.22-71.42, \mathrm{p}<0.01)$. A history of prior tumor resection, insular tumor location, and preoperative use of levetiracetam, phenytoin, and valproate were not statistically significantly associated with atypical speech sites $(\mathrm{p}=0.21,0.27$, $0.12,0.52$, and 0.17 , respectively; Table 3 ).

\section{Discussion}

In this study, we present stereotactic probabilistic maps of cortical speech arrest and anomia localization based on awake mapping in 102 craniotomy patients. Human speech localization by stimulation mapping has been extensively studied, beginning with the work of Penfield. The present investigation expands current knowledge by providing detailed probabilistic maps of cortical speech representation utilizing the accuracy of modern navigational stereotactic MRI. To our knowledge, this study is the first to report results based on stereotactic coordinates and in a probabilistic fashion-a format that will easily transition to a large, population-based probability map that can overlay onto an individual patient's MR image for the purposes of preoperative planning and risk stratification. Previous studies have created speech maps by clustering stimulation sites by gyri or into large "zones" based on intraoperative photographs and have not included confidence intervals on their reported probabilities. ${ }^{10,12,17,19}$ Here, probabilities were calculated in moving windows across the cortical surface to preserve the spatial resolution of the coordinates obtained from MRI navigation, and the standard deviation of this probability estimate was determined by statistical bootstrapping. We believe this probabilistic approach, accounting for variability, provides practical and intuitive maps of function that will prove helpful for neurosurgeons planning resections in and around eloquent speech areas. Further, the bootstrapped confidence intervals on the probability estimates in these maps give novel insight into the cortical areas with the greatest variability in speech localization between individuals. We identified areas of high speech arrest variability in the dorsal premotor cortex and the pSTG and high anomia variability in the posterior middle 

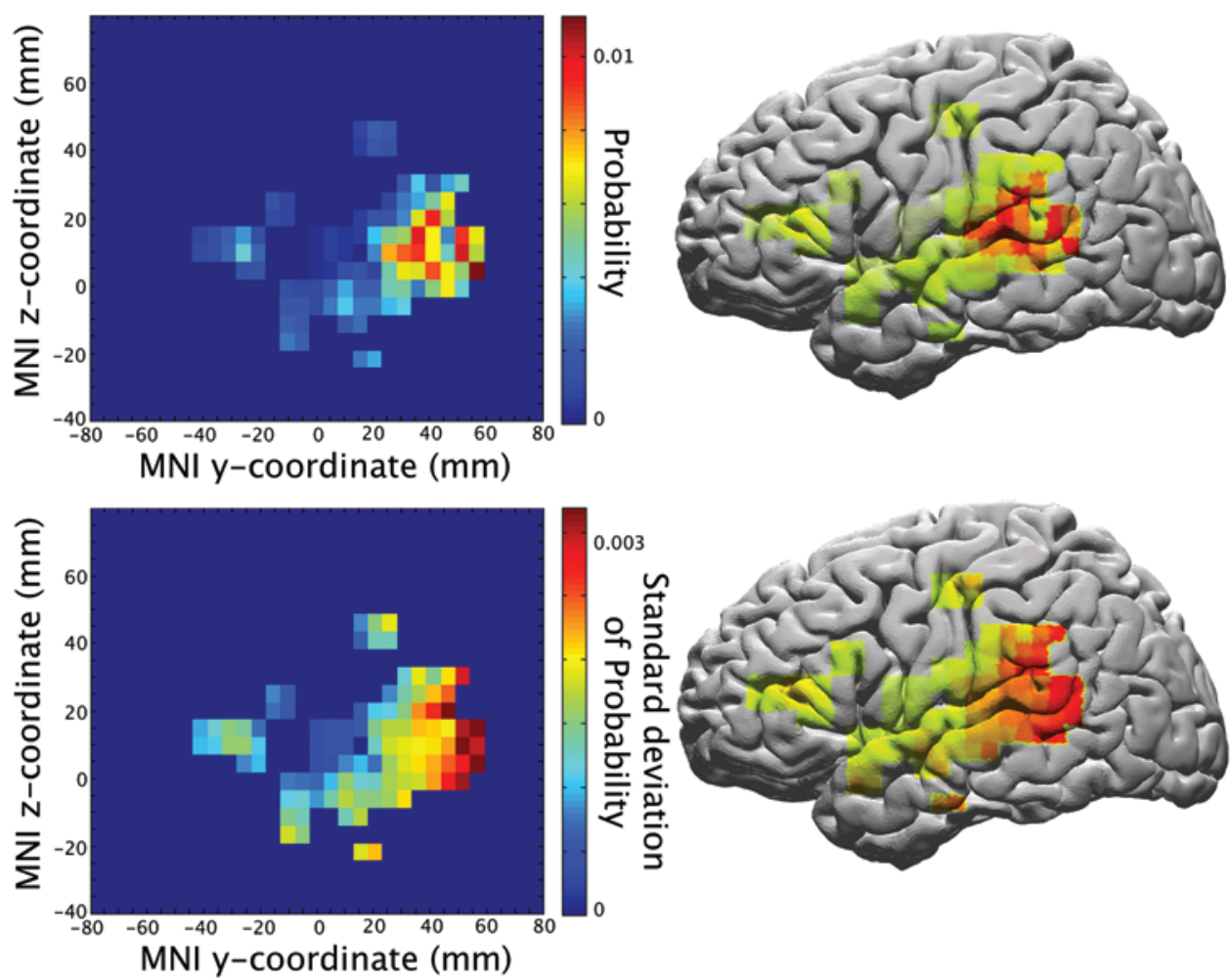

FIG. 3. Probability (upper) and variability (lower) of anomia sites. The pSTG demonstrated the highest probability of anomia, while the surrounding areas, including the posterior middle temporal, angular, and supramarginal gyri, showed the highest variability. Figure is available in color online only.

temporal, angular, and supramarginal gyri. Our multivariate analysis identified multilobar tumors and lesions in the canonical Wernicke's/Broca's areas as factors predictive of atypical language localization to these areas of greater variability. Duffau and colleagues reported bilateral gyrusbased probability maps of speech errors during stimulation mapping in awake craniotomies for the resection of lowgrade gliomas. ${ }^{19}$ They found $83 \%$ and $55 \%$ probabilities of speech arrest in the left and right ventral premotor cortex (vPMC), as well as $44 \%$ and $6 \%$ probabilities of anomia in the left and right STGs, respectively. Our results agree with the findings of Duffau's cluster analysis, and the "hotspots" for speech arrest and anomia functions seen in Figs. 2 and 3 , respectively. Like them, we demonstrated that stimulation-induced anomia tends to occur in the posterior temporal lobe, which is consistent with prior work. ${ }^{17}$

Speech arrest occurred with the highest probability during stimulation of the posterior frontal operculum, namely the pars triangularis, pars opercularis, and precentral gyrus in our patient population (Fig. 2), whereas the Tate et al. group found that stimulation of the precentral gyrus (especially the vPMC) led to the highest probability of anarthria. Prior cortical stimulation studies have shown that both canonical Broca's area and vPMC have been linked to the final common pathway for speech synthesis:, ${ }^{9}, 15,17,20,21$ thus, this discrepant localization may simply be a result of patient variability and selection bias. In fact, though speech arrest occurred frequently in the pars triangularis in our cohort, it was also a location of significant variability (SD 0.5\%; Fig. 2). Another possible explanation for this difference is that our probabilistic map used stereotactic neuronavigation to obtain the locations of each stimulation site, whereas the results presented by Tate et al. used radiographic landmarks, operative reports, and intraoperative photos for map generation. This methodological discrepancy in how sites were localized may account for the differing results obtained for the neighboring par opercularis and vPMC. We believe that the map described here accurately identifies the posterior frontal operculum, a portion of Broca's area, as essential for speech production. This appears to be supported by Ius et al., who showed unresectable areas in the par triangularis, pars opercularis, and $\mathrm{vPMC}$ of a "minimal common brain" created from postoperative MRI in 58 patients after low-grade glioma resection. ${ }^{9}$

In our population of patients, the dorsal premotor cortex and pSTG appeared to harbor the highest variability in speech arrest between individuals. Anomia seemed to be most variable in the posterior middle temporal, angular, and supramarginal gyri. Meanwhile, the areas of maximal probability for finding speech arrest and anomia are in the classic Broca's area and vPMC, and Wernicke's area, respectively. Not surprisingly, our multivariate analysis revealed that lesions involving one of these classic locations or involving more than 1 lobe of the brain were significantly associated with atypical localization of speech function. These findings suggest there is some potential for reorganization or spatial displacement of these classic speech areas and, again, are in agreement with Ius et al., who showed areas of intermediate resectability in the 
TABLE 2. Univariate analysis of variables potentially associated with atypical speech sites

\begin{tabular}{|c|c|c|c|}
\hline Predictor Variable & Classic Site Cohort $(n=86)$ & Atypical Site Cohort $(n=16)$ & p Value \\
\hline Median age in yrs & 40.5 & 47.8 & 0.24 \\
\hline Male sex, no. (\%) & $49(57)$ & $11(69)$ & 0.38 \\
\hline Prior tumor resection, no. $(\%)^{*}$ & $6 / 61(10)$ & $5 / 13(38)$ & 0.02 \\
\hline Lt-sided surgery, no. (\%) & $83(96)$ & $15(94)$ & 0.60 \\
\hline Mean tumor vol in $\mathrm{cm}^{3}( \pm$ SEM) & $50.3( \pm 51.5)$ & $32.6( \pm 39.0)$ & 0.29 \\
\hline High tumor grade, no. (\%)† & $51(59)$ & $10(62)$ & 0.85 \\
\hline History of seizures, no. (\%) & $60(70)$ & $9(56)$ & 0.29 \\
\hline \multicolumn{4}{|l|}{ Preop AED use, no. (\%) } \\
\hline Levetiracetam* & $12(14)$ & $5(31)$ & 0.08 \\
\hline Phenytoin* & $38(44)$ & $4(25)$ & 0.15 \\
\hline Valproic acid* & $3(3)$ & $3(19)$ & 0.02 \\
\hline Carbamazepine & $5(6)$ & $0(0)$ & 0.32 \\
\hline Oxcarbazepine & $3(3)$ & $0(0)$ & 0.45 \\
\hline Lamotrigine & $1(1)$ & $0(0)$ & 0.67 \\
\hline Topiramate & $2(2)$ & $1(6)$ & 0.39 \\
\hline Felbamate & $1(1)$ & $0(0)$ & 0.67 \\
\hline Multiple AEDs & $7(8)$ & $2(12)$ & 0.57 \\
\hline \multicolumn{4}{|l|}{ Tumor lobe, no. (\%) } \\
\hline Frontal only & $18(21)$ & $3(19)$ & 0.84 \\
\hline Temporal only & $23(27)$ & $5(31)$ & 0.71 \\
\hline Parietal only & $11(13)$ & $1(6)$ & 0.46 \\
\hline Insula only* & $28(32)$ & $1(6)$ & 0.03 \\
\hline Multiple lobes* & $6(7)$ & $6(38)$ & 0.001 \\
\hline Tumor in canonical Broca's/ Wernicke's areas, no. (\%)* & $14 / 62(22)$ & $8 / 13(62)$ & 0.02 \\
\hline
\end{tabular}

pars triangularis and pars opercularis. ${ }^{9}$ The variability and plasticity of the frontal operculum has also recently been highlighted by our recent work, which demonstrated only a $2.9 \%$ chance of new speech deficits after tumor resection in this area. ${ }^{16}$ Taken together, these results support the assertion that the classic model of speech has little predictive value for these patients and underscores the importance of tailoring surgical plans to individual patients. ${ }^{12}$

There are certainly limitations to the present study that deserve mention. First, the accuracy of probabilistic maps in achieving the goal of reflecting true probability in the general population is directly related to the number of

TABLE 3. Multivariate analysis of predictors of atypical speech sites

\begin{tabular}{lcc}
\hline \multicolumn{1}{c}{ Predictor Variable } & OR $(95 \% \mathrm{Cl})$ & p Value \\
\hline Prior tumor resection & $2.97(0.55-16.01)$ & 0.21 \\
\hline Preop AED use & & \\
$\quad$ Levetiracetam & $3.55(0.71-17.66)$ & 0.12 \\
$\quad$ Phenytoin & $0.54(0.09-3.44)$ & 0.52 \\
$\quad$ Valproic acid & $3.78(0.57-25.19)$ & 0.17 \\
\hline Tumor lobe & & \\
$\quad$ Insula only & $0.26(0.02-2.92)$ & 0.27 \\
$\quad$ Multiple lobes & $12.58(2.22-71.42)$ & 0.004 \\
\hline Tumor in canonical Broca's/ & $7.21(1.67-31.09)$ & 0.008 \\
$\quad$ Wernicke's areas & & \\
\hline
\end{tabular}

patients in the study population. Our study included 102 patients, and in order for this type of probabilistic map to be reliable enough for intraoperative use (as a coregistered overlay onto an individual patient's MR image, for example), we will have to continue accruing and incorporating data from more individuals. With more patients, a granular analysis of the effects of precise tumor location, grade, and size will be possible. In the present study, the classification of high versus low tumor grade and lobar location may have missed effects on language localization. Another important direction for future work will be to incorporate the results of noninvasive preoperative imaging modalities such as functional MRI and magnetoencephalography, which were not included in this study. Second, the cortical exposure for mapping individual patients is limited by the extent of their craniotomy. This may have resulted in the missed detection of speech sites; however, by combining data from 102 patients and employing a minimum threshold of 10 sites per bin for the inclusion of a cortical area in the map, we have ensured adequate coverage of the cortical areas relevant for surgical purposes.

\section{Conclusions}

The probabilistic maps presented here quantify the chances of inducing a speech arrest or anomia error at a given cortical location in an individual patient. They also quantify areas of increased variability in the location of these functions among individuals. We believe these maps 
will serve as an important tool for neurosurgeons treating lesions in eloquent areas.

\section{Acknowledgments}

Dr. Chang was funded by National Institutes of Health Grant Nos. R01-DC012379, R00-NS065120, and DP2-OD00862 and the Esther A. and Joseph Klingenstein Fund. E.F.C. is a New York Stem Cell Foundation Robertson Investigator. This research was supported by the New York Stem Cell Foundation.

\section{References}

1. Berger MS, Kincaid J, Ojemann GA, Lettich E: Brain mapping techniques to maximize resection, safety, and seizure control in children with brain tumors. Neurosurgery 25:786-792, 1989

2. Berger MS, Rostomily RC: Low grade gliomas: functional mapping resection strategies, extent of resection, and outcome. J Neurooncol 34:85-101, 1997

3. Burchiel KJ, Clarke H, Ojemann GA, Dacey RG, Winn HR: Use of stimulation mapping and corticography in the excision of arteriovenous malformations in sensorimotor and language-related neocortex. Neurosurgery 24:322-327, 1989

4. Chang EF, Clark A, Smith JS, Polley MY, Chang SM, Barbaro NM, et al: Functional mapping-guided resection of low-grade gliomas in eloquent areas of the brain: improvement of long-term survival. Clinical article. J Neurosurg 114:566-573, 2011

5. Chang EF, Wang DD, Perry DW, Barbaro NM, Berger MS: Homotopic organization of essential language sites in right and bilateral cerebral hemispheric dominance. J Neurosurg 114:893-902, 2011

6. De Witt Hamer PC, Moritz-Gasser S, Gatignol P, Duffau $\mathrm{H}$ : Is the human left middle longitudinal fascicle essential for language? A brain electrostimulation study. Hum Brain Mapp 32:962-973, 2011

7. Duffau H, Moritz-Gasser S, Gatignol P: Functional outcome after language mapping for insular World Health Organization Grade II gliomas in the dominant hemisphere: experience with 24 patients. Neurosurg Focus 27(2):E7, 2009

8. Haglund MM, Berger MS, Shamseldin M, Lettich E, Ojemann GA: Cortical localization of temporal lobe language sites in patients with gliomas. Neurosurgery 34:567-576, 1994

9. Ius T, Angelini E, Thiebaut de Schotten M, Mandonnet E, Duffau H: Evidence for potentials and limitations of brain plasticity using an atlas of functional resectability of WHO grade II gliomas: towards a "minimal common brain". Neuroimage 56:992-1000, 2011

10. Ojemann G, Ojemann J, Lettich E, Berger M: Cortical language localization in left, dominant hemisphere. An electrical stimulation mapping investigation in 117 patients. J Neurosurg 71:316-326, 1989

11. Ojemann GA: Brain organization for language from the perspective of electrical stimulation mapping. Behav Brain Sci 6:189-206, 1983

12. Ojemann GA: Individual variability in cortical localization of language. J Neurosurg 50:164-169, 1979

13. Ojemann SG, Berger MS, Lettich E, Ojemann GA: Localization of language function in children: results of electrical stimulation mapping. J Neurosurg 98:465-470, 2003
14. Penfield W, Roberts L: Speech and Brain Mechanisms. Princeton, NJ: Princeton University Press, 1959

15. Quiñones-Hinojosa A, Ojemann SG, Sanai N, Dillon WP, Berger MS: Preoperative correlation of intraoperative cortical mapping with magnetic resonance imaging landmarks to predict localization of the Broca area. J Neurosurg 99:311-318, 2003

16. Rolston JD, Englot DJ, Benet A, Li J, Cha S, Berger MS: Frontal operculum gliomas: language outcome following resection. J Neurosurg 122:725-734, 2015

17. Sanai N, Mirzadeh Z, Berger MS: Functional outcome after language mapping for glioma resection. N Engl J Med 358:18-27, 2008

18. Schwartz TH, Devinsky O, Doyle W, Perrine K: Functionspecific high-probability "nodes" identified in posterior language cortex. Epilepsia 40:575-583, 1999

19. Tate MC, Herbet G, Moritz-Gasser S, Tate JE, Duffau H: Probabilistic map of critical functional regions of the human cerebral cortex: Broca's area revisited. Brain 137:27732782,2014

20. van Geemen K, Herbet G, Moritz-Gasser S, Duffau H: Limited plastic potential of the left ventral premotor cortex in speech articulation: evidence from intraoperative awake mapping in glioma patients. Hum Brain Mapp 35:1587-1596, 2014

21. Wu J, Lu J, Zhang H, Zhang J, Mao Y, Zhou L: Probabilistic map of language regions: challenge and implication. Brain 138:e337, 2015

22. Yordanova YN, Moritz-Gasser S, Duffau H: Awake surgery for WHO Grade II gliomas within "noneloquent" areas in the left dominant hemisphere: toward a "supratotal" resection. Clinical article. J Neurosurg 115:232-239, 2011

\section{Disclosures}

The authors report no conflict of interest concerning the materials or methods used in this study or the findings specified in this paper.

\section{Author Contributions}

Conception and design: Chang, Breshears, Raygor, Berger. Acquisition of data: Chang, Breshears, Raygor, Lau. Analysis and interpretation of data: Chang, Breshears, Raygor. Drafting the article: Chang, Breshears, Raygor. Critically revising the article: Chang, Breshears, Raygor, Berger. Reviewed submitted version of manuscript: Chang, Breshears, Molinaro, Berger. Statistical analysis: Breshears, Raygor, Molinaro. Study supervision: Chang, Berger.

\section{Supplemental Information \\ Previous Presentations}

Portions of this work were presented in poster form at the Congress of Neurological Surgeons Annual Meeting held in Boston, Massachusetts, on October 18-22, 2014.

\section{Correspondence}

Edward F. Chang, Department of Neurological Surgery, University of California, San Francisco, 505 Parnassus Ave., M-779, San Francisco, CA 94143-0112. email: changed@ neurosurg.ucsf.edu. 